\title{
Icariin promotes osteogenic differentiation of BMSCs by upregulating BMAL1 expression via BMP signaling
}

\author{
ZENGFA HUANG $^{1 *}$, HUI WEI $^{2 *}$, XIANG WANG $^{1}$, JIANWEI XIAO $^{1}$, ZUOQIN LI $^{1}$, \\ YUANLIANG XIE ${ }^{1}$, YUN HU ${ }^{1}$, XIANG LI $^{1}$, ZHENG WANG $^{1}$ and SHUTONG ZHANG ${ }^{1}$ \\ ${ }^{1}$ Department of Radiology, The Central Hospital of Wuhan, Tongji Medical College, \\ Huazhong University of Science and Technology, Wuhan, Hubei 430014; ${ }^{2}$ Department of Orthopedics, \\ The Affiliated Changzhou No. 2 People's Hospital of Nanjing Medical University, Changzhou, Jiangsu 213003, P.R. China
}

Received June 18, 2019; Accepted December 3, 2019

DOI: $10.3892 / \mathrm{mmr} .2020 .10954$

\begin{abstract}
Increasing research has demonstrated that expression of brain and muscle ARNT-like 1 (BMAL1) and other circadian clock genes can be regulated by drugs and toxicants. We previously demonstrated that icariin, extracted from Herba Epimedii, sromotes osteogenic differentiation. However, the mechanism underlying the association between icariin and BMAL1 in osteogenic differentiation of bone marrow-derived mesenchymal stem cells (BMSCs) remains unclear. The present study was designed with an aim to clarify the association between icariin and BMAL1 in osteogenic differentiation of BMSCs. The Cell Counting Kit- 8 assay was used to evaluate cell proliferation. The expression of bone morphogenetic protein 2 (BMP2), RUNX family transcription factor 2 (RUNX2), alkaline phosphatase (ALP), osteocalcin (OC) and BMAL1 in BMSCs was evaluated by reverse transcription-quantitative PCR and western blotting. ALP and Alizarin red S (ARS) staining were also performed. Icariin promoted BMSC proliferation, and upregulated expression of osteogenic genes and BMAL1. In addition, expression of the osteogenic genes BMP2, RUNX2, ALP and OC were upregulated by BMAL1 overexpression. Furthermore, we confirmed that BMAL1 deficiency suppressed osteogenic differentiation in BMSCs. Finally, ARS staining of BMAL1 ${ }^{-1-}$ BMSCs revealed that BMAL1 was an essential intermediary in matrix mineralization during osteogenic differentiation. In conclusion, these results demonstrated that icariin promoted
\end{abstract}

Correspondence to: Dr Shutong Zhang, Department of Radiology, The Central Hospital of Wuhan, Tongji Medical College, Huazhong University of Science and Technology, 26 Shengli Avenue, Jiangan, Wuhan, Hubei 430014, P.R. China

E-mail: zhangshutong1960@sina.com

${ }^{*}$ Contributed equally

Key words: icariin, bone marrow-derived mesenchymal stem cells, brain and muscle ARNT-like 1, bone morphogenetic protein 2, osteogenic differentiation osteogenic differentiation through BMAL1-BMP2 signaling in BMSCs. The present study thus described a novel target of icariin that has potential applications in the treatment of osteogenic disorders.

\section{Introduction}

Osteonecrosis of the femoral head (ONFH) is a debilitating and progressive disease which can lead to femoral head collapse and subsequent osteoarthritis. This disease is the major cause of total hip arthroplasty in young adults (1). It is reported that more than 1 million new patients are affected with this disease annually and the annual incidence is 15 to 20 million according to a nationwide survey (2). Accumulating evidence has alluded to several etiologic and pathogenic mechanisms for ONFH (3). The principal mechanism involves interruption of osteogenic differentiation of bone marrow-derived mesenchymal stem cells (BMSCs) (4). Thus, investigation of the disordered mechanism of osteogenic differentiation in BMSCs and development of effective methods of prevention and early therapy are crucial.

A number of previous studies have reported that osteogenic differentiation-related genes are expressed periodically $(5,6)$. Brain and muscle ARNT-like 1 (BMAL1) is the most important component of the molecular biological clock, expression of which has been found to have 24-h periodicity in bone. The circadian system rhythmically regulates cellular proliferation, physiology, and behavior $(7,8)$. Moreover, it is implicated in drug efficacy, metabolism, detoxification and toxicity (9-11). The core transcriptional activator of the clock network is BMAL1 which is regulated by delicate systems in mammals (7). Increasing numbers of studies have demonstrated that BMAL1 and other circadian clock genes could be regulated by drugs and toxicants. Miranda et al (12) showed that resveratrol could reverse circadian disruption induced by a high-fat diet in rats. Chen et al (13) demonstrated that carbon tetrachloride altered circadian rhythms of liver clock genes in a mouse model of hepatic fibrosis, while Gabás-Rivera et al (14) reported that dietary oleanolic acid supplementation mediated circadian clock gene expression in an animal model.

Icariin is extracted from Herba Epimedii which is widely used as a major active ingredient to prevent osteonecrosis 
induced by glucocorticoids in patients with severe acute respiratory syndrome. Our previous study revealed that icariin regulated cell proliferation and osteogenic differentiation in MC3T3-E1 cells (15) and acted as an effective ingredient for preventing the progression of ONFH induced by glucocorticoids in a rat model (16). However, the mechanism underlying the association between icariin and BMAL1 in osteogenic differentiation of BMSCs remains unclear.

In the present study, it was demonstrated that icariin could alter BMAL1 and induce osteogenic differentiation of BMSCs in vivo. Furthermore, it was also shown that icariin enhanced the bone morphogenetic protein 2 (BMP2)/RUNX family transcription factor 2 (RUNX2) signaling pathway through upregulation of BMAL1 expression.

\section{Materials and methods}

Cell culture and treatment. According to the method described by Gong et al (17), a total of 20 3-week-old female Sprague-Dawley rats (Laboratory Animal Center of Huazhong University of Science and Technology) were sacrificed by the intraperitoneal injection of $100 \mathrm{mg} / \mathrm{kg}$ sodium pentobarbital and BMSCs were extracted from femurs and tibias by aseptic manipulation. Cells were expanded in minimum essential medium ( $\alpha$-MEM; Gibco; Thermo Fisher Scientific, Inc.) containing $10 \%$ fetal bovine serum (FBS; Invitrogen; Thermo Fisher Scientific, Inc.) and $1 \%$ penicillin G-streptomycin and cultured in $5 \% \mathrm{CO} 2$ at $37^{\circ} \mathrm{C}$. BMSCs at a density of $80-90 \%$ were passaged and cells at passage three to six were used in the following experiments. For osteogenic induction, basic medium was supplemented with $10^{-7} \mathrm{~mol} / \mathrm{l}$ dexamethasone, $10^{-2} \mathrm{~mol} / \mathrm{l}$ sodium $\beta$-glycerophosphate and $50 \mu \mathrm{g} / \mathrm{ml} \mathrm{L}$-ascorbic acid. The medium was changed every 3 days for BMSC differentiation. Each experiment was performed in triplicate. All the experimental protocols involving animals were approved by the Institutional Animal Care and Use Committee of Tongji Medical College, Huazhong University of Science and Technology (IACUU no. S496; date of permission, October, 31, 2017).

Icariin (purity $>98 \%$; Abcam) was dissolved in dimethyl sulfoxide (DMSO) and then stored at $-20^{\circ} \mathrm{C}$ without light. Subsequent experiments used a DMSO concentration of $\leq 0.1 \%$. All experiments were performed in triplicate.

Cell Counting Kit-8 (CCK-8) assay. The effect of icariin on cell proliferation was evaluated using the CCK-8 assay. Briefly, BMSCs $\left(5 \times 10^{3}\right.$ cells/well) were plated in 96-well plates and treated with icariin $\left(10^{-10}-10^{-3} \mathrm{~mol} / \mathrm{l}\right)$ for $48 \mathrm{~h}$ at $37^{\circ} \mathrm{C}$. Subsequently, cell proliferation was assessed using the CCK-8 reagent (Nanjing Jiancheng Bioengineering Institute), according to the manufacturer's instructions, at a wavelength of $450 \mathrm{~nm}$ using an ELx800 multifunctional microplate reader (BioTek Instruments, Inc.). The concentration of icariin used to treat the cells that were analyzed by RT-qPCR and western blotting was based on the results of the CCK-8 assay.

Flow cytometry. After reaching confluence, BMSCs at passage 3-4 were used for flow cytometric analysis. First, the cells were incubated with trypsin for $1 \mathrm{~min}$ at $37^{\circ} \mathrm{C}$, and then the cell pellet was collected by transient centrifugation at $400 \mathrm{x} \mathrm{g}$ at room temperature for $5 \mathrm{~min}$. Next, the cell pellet was washed with ice-cold PBS and then centrifuged at $400 \mathrm{x} \mathrm{g}$ at room temperature for $5 \mathrm{~min}$. Subsequently, the cell pellet was resuspended in $100 \mu 1$ ice-cold PBS supplemented with $0.5 \%$ BSA (Invitrogen; Thermo Fisher Scientific, Inc.) for $20 \mathrm{~min}$ at $4^{\circ} \mathrm{C}$. Additionally, the cells were treated with Rat Fc block (cat. no. 550271; BioLegend, Inc.) for $20 \mathrm{~min}$ at $4^{\circ} \mathrm{C}$. Finally, the cells were identified by negative expression of CD34 [phycoerythrin (PE)-labeled; cat. no. 128611; BioLegend, Inc.] and CD45 (FITC-labeled; cat. no. 202205; BioLegend, Inc.) and positive expression of CD73 (FITC-labeled; cat. no. 127219; BioLegend, Inc.), CD90 (PE-labeled; cat. no. 205903; BioLegend, Inc.) and CD105 (PE-labeled; cat. no. 120407; BioLegend, Inc.) using a FACSscan flow cytometer (Beckton Dickinson) and analyzed by FlowJo software (version X; FlowJo LLC).

Reverse transcription-quantitative PCR (RT-qPCR). Total RNA was extracted with TRIzol (Invitrogen; Thermo Fisher Scientific, Inc.) from BMSCs and the reverse transcription RT reaction (starting with $1 \mu \mathrm{g}$ of total RNA) was performed using the EasyScript One-Step gDNA Removal and cDNA Synthesis Supermix (Beijing TransGen Biotech Co., Ltd.) according to the manufacturer's instructions. The SYBR Green/ROX RT-qPCR mix (Takara Bio, Inc.) and the StepOnePlus ${ }^{\mathrm{TM}}$ Real-Time PCR System (Applied Biosystems; Thermo Fisher Scientific, Inc.) were used for mRNA quantification, according to the manufacturer's protocol. The primer sequences used for PCR were as follows: BMAL1 forward, 5'-AACCTTCCCGCAGCT AACAG-3' and reverse, 5'-AGTCCTCTTTGGGCCACCTT-3'; BMAL1 $^{-/}$, forward 5'-CCACCAAGCCCAGCAACTCA-3' and reverse, 5'-TCGCCTTCTATGGCCTTCTTGACG-3'; BMAL1 (overexpression) forward, 5'-AGGTCGACTCTAGAGGAT CCCGCCACCATGGCGGACCAGAGAATGGACATTTC-3' and reverse, 5'-TCCTTGTAGTCCATACCCAGCGGCCAT GGCAAGTCACTAAAG-3'; BMP2 forward, 5'-AGGATG CTGGGAAGTCCG-3' and reverse, 5'-AGGTGCCACGAT CCAGTCAT-3'; RUNX2, forward, 5'-AGCGGACGAGGC AAGAGTTT-3' and reverse, 5'-AGGCGGGACACCTACTCT CATA-3'; alkaline phosphatase (ALP) forward, 5'-CAAGGA TGCTGGGAAGTCCG-3' and reverse, 5'-CTCTGGGCGCAT CTCATTGT-3'; osteocalcin (OC) forward, 5'-TCAACAATG GACTTGGAGCCC-3' and reverse, 5'-AGCTCGTCACAATTG GGGTT-3'; and $\beta$-actin forward, 5'-GAGACCTTCAACACC CCAGC-3' and reverse, 5'-ATGTCACGCACGATTTCCC-3'. The reaction conditions were as follows: $95^{\circ} \mathrm{C}$ for $15 \mathrm{sec}, 60^{\circ} \mathrm{C}$ for $20 \mathrm{sec}$ and $75^{\circ} \mathrm{C}$ for $10 \mathrm{sec}$ after denaturing DNA templates at $95^{\circ} \mathrm{C}$ for $10 \mathrm{~min} ; 40$ cycles. The $2^{-\Delta \Delta \mathrm{Cq}}$ method (18) was used to determine the relative expression levels.

Western blotting. Total protein was extracted with RIPA buffer (Sigma-Aldrich; Thermo Fisher Scientific, Inc.) according to standard protocols. Cell lysates with $50 \mu \mathrm{g}$ of total protein (quantified using the bicinchoninic acid assay) were separated by $10 \%$ SDS-PAGE, transferred onto PVDF membranes, and then blocked in TBST with 5\% non-fat dried milk for $1 \mathrm{~h}$ at room temperature. The membranes were incubated with primary monoclonal mouse antibodies targeted against: BMAL1 (1:1,000; cat. no. ab93806; Abcam), BMP2 (1:1,000; cat. no. ab14933; Abcam), RUNX2 (1:1,000; cat. no. ab23981; Abcam), ALP (1:1,000; cat. no. ab95462; Abcam), OC (1:1,000; 

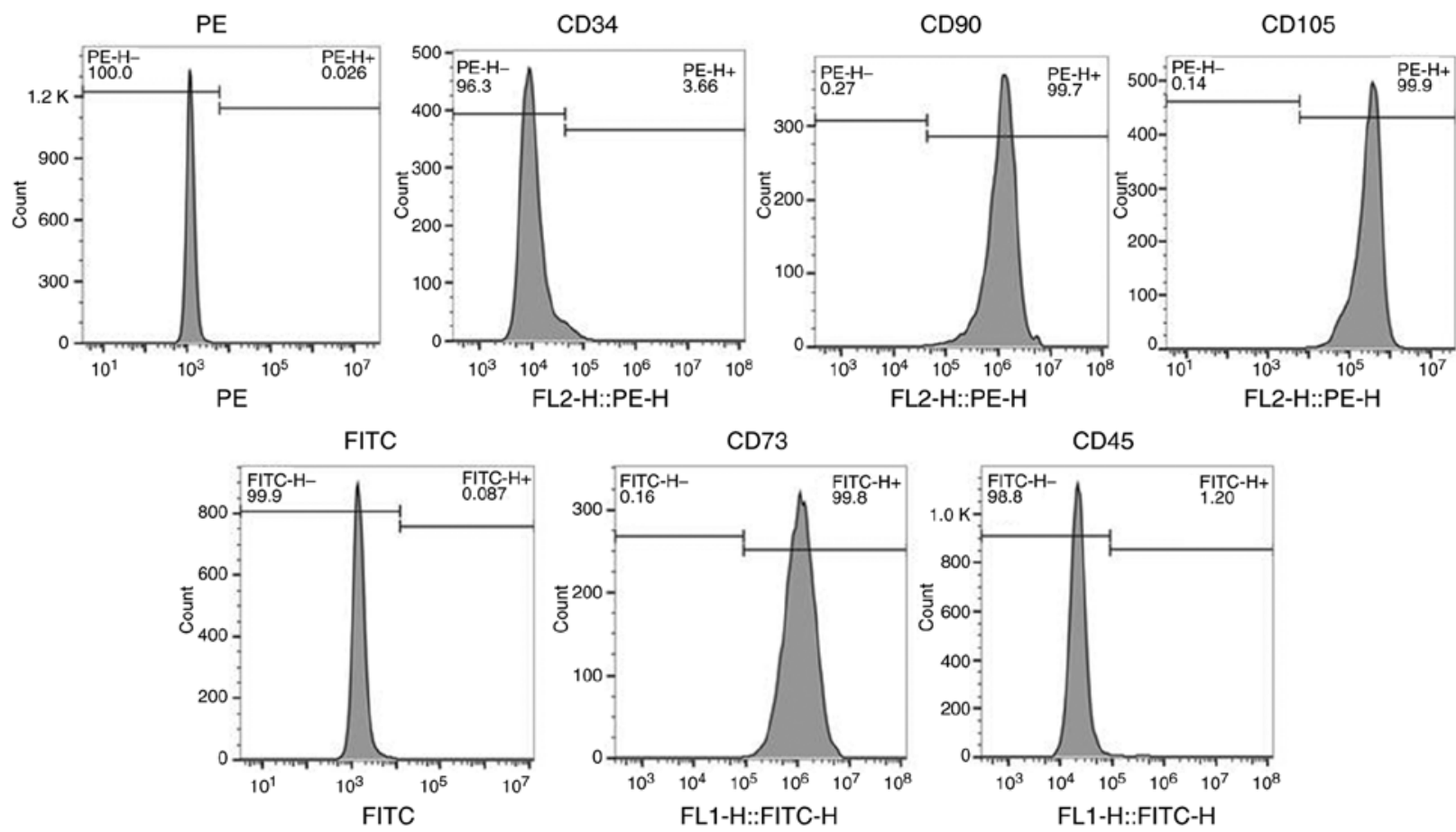

Figure 1. Flow cytometric analysis of cell surface markers of BMSCs. BMSCs were stained for cell surface markers. BMSCs were largely positive for CD73, CD90, CD105 and negative for CD34, CD45. BMSCs, bone marrow-derived mesenchymal stem cells; CD, cluster of differentiation.

cat. no. ab34710; Abcam) and $\beta$-actin (1:1,000; cat. no. ab8227; Abcam) at $4^{\circ} \mathrm{C}$ overnight, and then followed by secondary antibodies conjugated with horseradish peroxidase $(1: 10,000$; cat. no. ASP00001; Dako; Agilent Technologies, Inc.) for $1 \mathrm{~h}$ at room temperature after washing. The immunoblots were visualized and detected using the ECL system (Pierce; Thermo Fisher Scientific, Inc.). Protein expression was quantified using Fusion Solo software (version 4; Vilber Lourmat Deutschland $\mathrm{GmbH})$.

Transient transfection and luciferase assay. BMAL1 short hairpin RNA (shRNA) interference lentiviral vector was constructed and synthesized by Shanghai Genechem Co., Ltd. The BMAL1 shRNA interference target sequence was 5'-ACA CGCAATAGATGGGAAA-3', and a scramble sequence 5'-TTCAAGATCCTCAATTATA-3' was used as a negative control. BMSCs were cultured in 6-well plates with specific media to appropriate confluence and then transfected with the virus assisted with $6 \mu \mathrm{g} / \mathrm{ml}$ polybrene for $12 \mathrm{~h}$ at $37^{\circ} \mathrm{C}$. The viral supernatants were removed and the culture was continued in complete medium. BMSCs were grown to $30-70 \%$ confluence and then transfected with BMAL1, BMAL1-shRNAs or empty vector overnight. The following day, the cells were co-transfected with pRL-SV40 Renilla luciferase reporter plasmids (10 ng; Promega Corporation) and firefly luciferase reporter vectors (50 ng), using Lipofectamine ${ }^{\circledR} 2000$ (Invitrogen; Thermo Fisher Scientific, Inc.) as the delivery system and a control. Cells were lysed after a 48-h transfection and firefly and Renilla luciferase activities were measured using the Dual Luciferase Reporter Assay system (Promega Corporation).

ALP and Alizarin red $S(A R S)$ staining. BMSCs were seeded at a density of $1 \times 10^{5}$ cells/well in 6-well plates and incubated with osteogenic medium. According to the manufacturer's instructions, ALP staining was assessed using an ALP staining kit (Nanjing Jiancheng Bioengineering Institute) on day 7 .

After osteogenic induction for 14 days, the cells were washed three times with PBS and fixed in $4 \%$ paraformaldehyde for $30 \mathrm{~min}$ at room temperature. Then paraformaldehyde was removed and the cells were washed three times with $\mathrm{ddH}_{2} \mathrm{O}$. Finally, the cells were stained with $0.04 \mathrm{M}$ ARS for $30 \mathrm{~min}$ at room temperature, and then rinsed twice with $\mathrm{dd}_{2} \mathrm{O}$ and visualized under a light microscope (magnification, $\mathrm{x} 40$ ). For quantitative analysis of ARS staining, the absorbance at $540 \mathrm{~nm}$ was measured using a UV spectrophotometer. Results of ARS are expressed as $\mu \mathrm{g} / \mathrm{mg}$ protein with average and standard deviation.

Statistical analysis. Data are presented as the mean \pm SD. Statistical analyses were performed using SPSS software (version 17.0; SPSS, Inc.). Differences between groups were analyzed using an unpaired Student's t-test or one-way ANOVA followed by Dunnett's post hoc test. $\mathrm{P}<0.05$ was considered to indicate statistical significance.

\section{Results}

Identification of BMSCs using stem cell markers. Flow cytometry was performed to identify BMSCs by expression of CD34, CD45, CD73, CD90 and CD105 in the isolated cells. The percentages of CD73-positive cells, CD90-positive cells and CD105-positive cells were 99.8, 99.7 and 99.9\%, respectively; while the percentages of CD34-positive cells and CD45-positive cells were 3.66 and $1.2 \%$, respectively, indicating that the isolated cells were BMSCs (Fig. 1). 

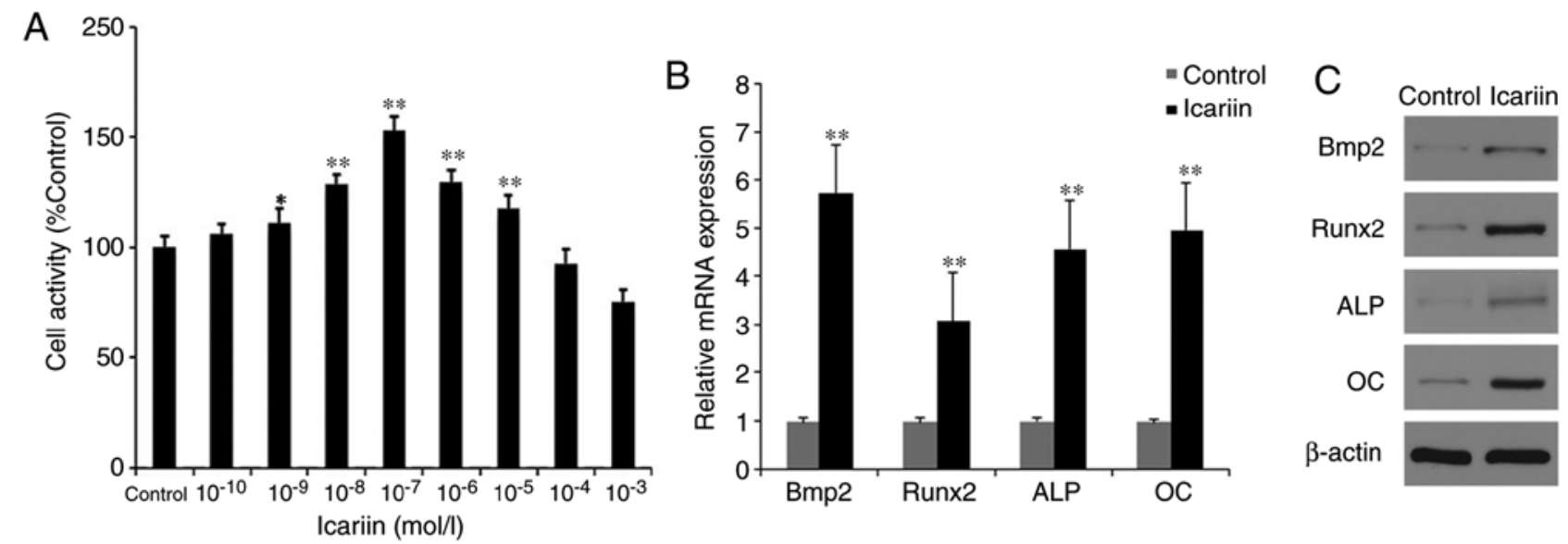

Figure 2. Icariin promotes cell proliferation activity and expression of osteogenic genes in BMSCs. (A) BMSCs were treated with icariin $\left(10^{-10}-10^{-3} \mathrm{M}\right)$ for $48 \mathrm{~h}$ and assessed by the Cell Counting Kit-8 assay. (B) mRNA expression and (C) protein level of osteogenic genes in control and $10^{-7} \mathrm{M}$ icariin-treated groups Data are expressed as mean $\pm \mathrm{SD} .{ }^{*} \mathrm{P}<0.05$ and ${ }^{* *} \mathrm{P}<0.01$ (compared with the control); by one-way ANOVA followed by Dunnett's post hoc test or the Student's t-test. BMSCs, bone marrow-derived mesenchymal stem cells; BMP2, bone morphogenetic protein 2; RUNX2, RUNX family transcription factor 2; ALP, alkaline phosphatase; OC, osteocalcin.

Icariin induces expression of osteoblastic genes in BMSCs. The proliferative activity of BMSCs in the presence of different concentrations of icariin $\left(10^{-10}-10^{-3} \mathrm{M}\right)$ was determined using the CCK-8 assay (Fig. 2A). The results showed that cell proliferation was significantly increased by lower concentrations of icariin $\left(10^{-9}-10^{-5} \mathrm{M} ; \mathrm{P}<0.05\right)$, with $10^{-7} \mathrm{M}$ being the most effective.

RT-qPCR and western blotting demonstrated that icariin significantly increased the expression of BMP2, RUNX2, ALP and $\mathrm{OC}$ (Fig. 2B and C).

Icariin regulates $B M A L 1$ in BMSCs. To investigate whether icariin induces BMAL1 expression in BMSCs, BMAL1 expression of $10^{-7} \mathrm{M}$ icariin-treated cells was evaluated by RT-qPCR and western blotting. Icariin significantly increased BMAL1 mRNA and protein levels (Fig. 3). These results suggested that icariin induced BMAL1 expression in BMSCs.

BMAL1 induces osteogenic differentiation via BMP2 in $B M S C s$. To evaluate whether BMAL1 regulates osteogenic differentiation via BMP2, BMSCs were transduced with BMAL1. BMAL1 overexpression increased expression of osteogenic differentiation-associated genes. Moreover, BMAL1 overexpression enhanced both mRNA expression and the promoter activity of BMP2 (Fig. 4E and F).

BMAL1 deficiency suppresses osteogenic differentiation in $B M S C$ s. In order to determine whether BMAL1 is essential for osteogenic differentiation induced by icariin, the osteogenic differentiation-inducing effects of $10^{-7} \mathrm{M}$ icariin treatment were further investigated in BMAL1-knockdown (BMAL1 ${ }^{-1}$ ) BMSCs. Compared with the effects of icariin in wild-type (WT) BMSCs, expression of osteogenic genes was reduced in the BMAL1 ${ }^{-1-}$ BMSCs evaluated by PCR analysis (Fig. 4E). In accordance with the PCR results, western blotting also showed that levels of BMP2, RUNX2, ALP and OC were lower in the BMAL1 ${ }^{-1}$ BMSCs compared to the WT BMSCs (Fig. 4F). Furthermore, ARS of BMAL1 $1^{-/}$BMSCs indicated that BMAL1 was required for mineralized nodule formation

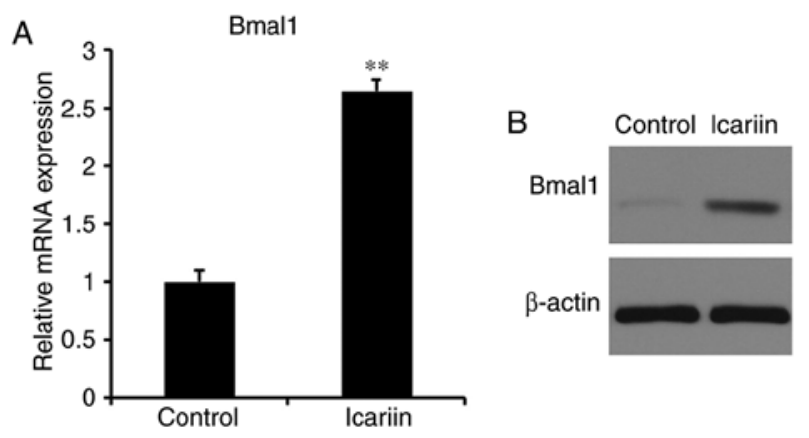

Figure 3. Icariin increases BMAL1 expression in BMSCs. (A) mRNA expression of BMAL1. (B) Protein level of BMAL1 as assessed by western blot analysis. ${ }^{* *} \mathrm{P}<0.01$ (compared with control). BMSCs, bone marrow-derived mesenchymal stem cells; BMAL1, brain and muscle ARNT-like 1.

during osteogenic differentiation (Fig. 4B and D). In addition, less positive staining was detected in $\mathrm{BMAL1}^{-/} \mathrm{BMSCs}$ (Fig. 4A and C).

Taken together, icariin increased the BMAL1, BMP2 and RUNX2 expression in BMSCs. BMAL1 overexpression also upregulated BMP2 and RUNX2 expression compared with the control group. However, these levels of expression were significantly downregulated after BMAL1 knockdown whether icariin treatment was administered or not. The results suggested the icariin could enhance the BMP2 signaling pathway by upregulating BMAL1 expression in vitro.

\section{Discussion}

In the present study, it was demonstrated that icariin promoted osteogenic differentiation by upregulating BMAL1 expression through BMP signaling in BMSCs. These findings suggested that BMAL1 plays a critical role in icariin-mediated osteogenic differentiation.

In fact, a circadian clock exists in every cell of the human body. The central players in these are BMAL1 encoded by the gene ARNTL and clock circadian regulator (CLOCK) 
A
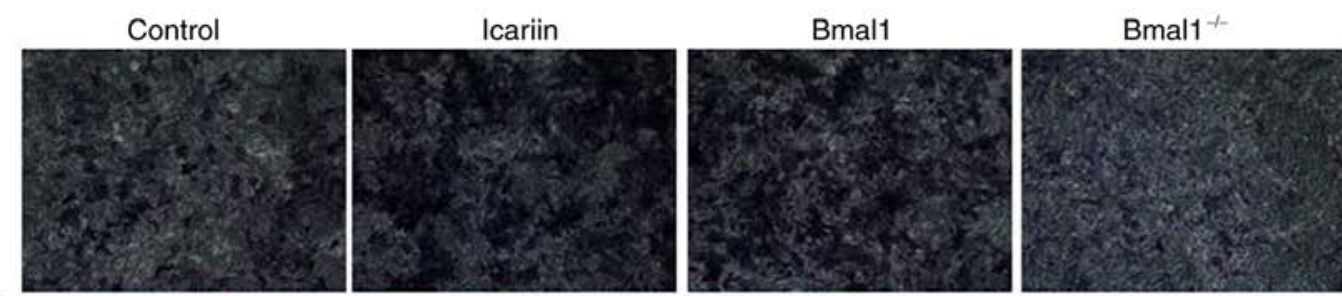

Icariin+Bmal1 ${ }^{+1}$

B
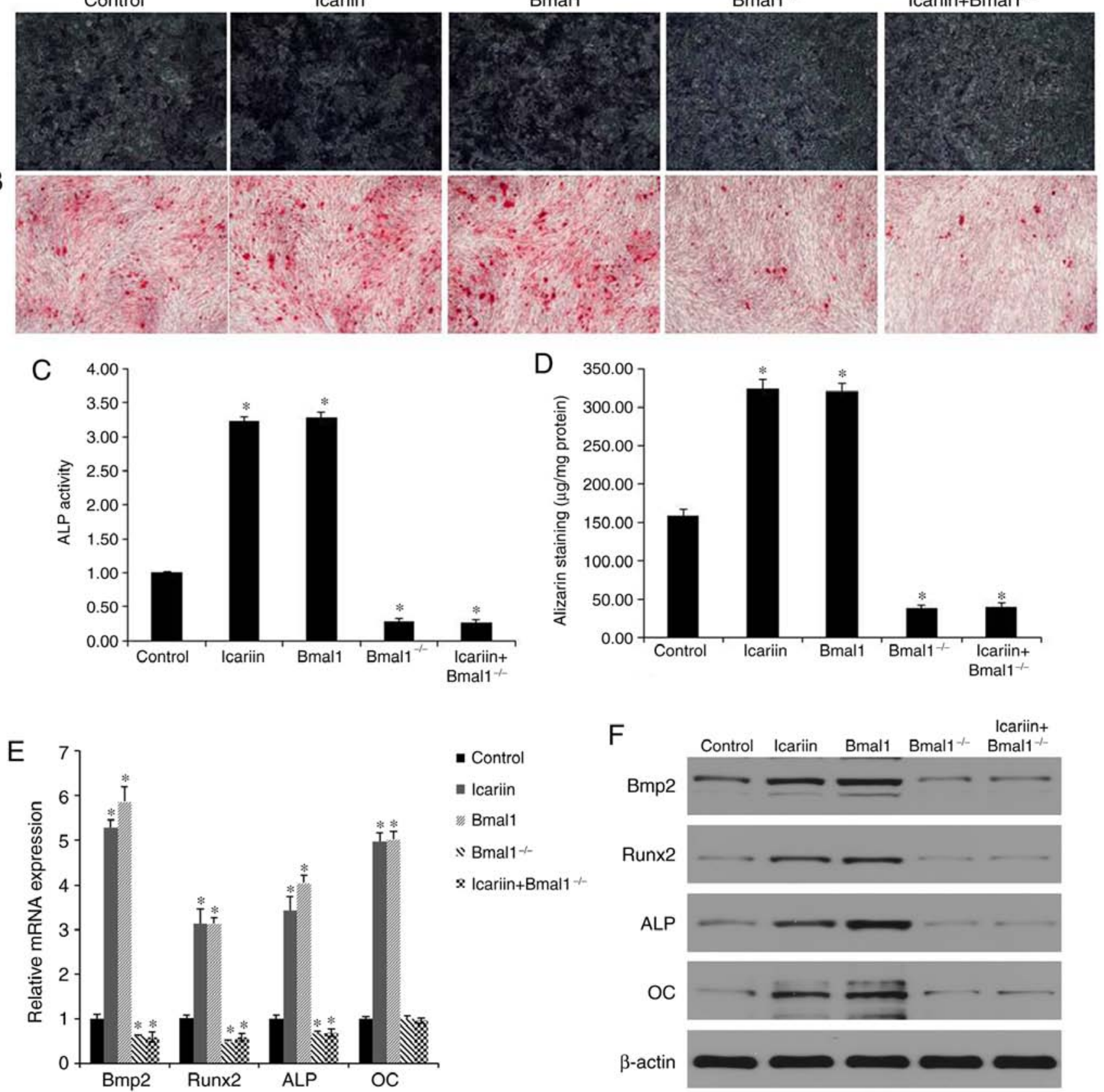

$\mathrm{F}$

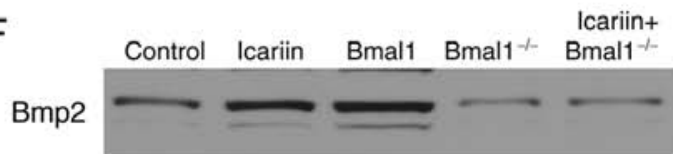

Runx2
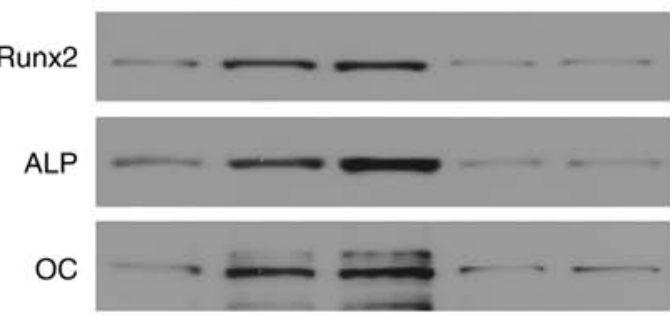

$\beta$-actin

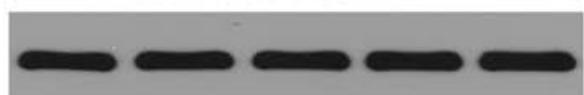

Figure 4. Osteogenic differentiation of BMSCs with BMAL1 overexpression (BMAL1) and BMAL1 deficiency (BMAL1 ${ }^{-/}$). (A) ALP staining. (B) ARS staining performed to examine extracellular mineralization. (C) Quantitative ALP analysis. (D) Quantitative ARS staining analysis. (E) Reverse transcription-quantitative PCR and (F) western blot analysis of osteogenic gene expression in BMSCs. "P<0.05 (compared with the control) by one-way ANOVA followed by Dunnett's post hoc test. BMSCs, bone marrow-derived mesenchymal stem cells; BMAL1, brain and muscle ARNT-like 1; BMP2, bone morphogenetic protein 2; RUNX2, RUNX family transcription factor 2; ALP, alkaline phosphatase; OC, osteocalcin; ARS, alizarin red S.

by the gene CLOCK (19). BMSCs are stem cells with the ability to differentiate in vitro into adipocytes, osteoblasts and cartilage-forming chondroblasts (20). Regardless of its exact developmental origin, the function of the circadian clock gene is related to the behavior of various stem cells involved in homeostasis and repair of bone and adipose tissue (21). A recent report revealed impairment of osteogenic differentiation of BMSCs from BMAL1 ${ }^{-/}$mice (5). This finding was in line with our present study as well as an observational study that showed that the numbers of active osteocytes and osteoblasts were reduced in $\mathrm{BMAL1}^{-/}$mice in vivo (22). However, a previous study showed that the amount of osteoblastic activity was increased in $\mathrm{BMAL1}^{-/-}$mice (21). These differences may due to the different ages of the mice. In this study, we clearly evaluated the positive regulatory mechanism of BMAL1 on osteogenic differentiation. Our results indicated that BMAL1 could accelerate osteogenic differentiation through BMP2 signaling. BMP2, a member of the transforming growth factor (TGF)- $\beta$ superfamily, is considered to be a key regulator of cell growth and differentiation (23-25). A number of studies have suggested that the activity of clock genes is closely correlated with BMPs. One study reported that regulation of clock genes controlled BMP2 expression in osteoblasts (26). Meanwhile, BMAL1 regulation of TGF- $\beta$ and BMP signaling in the control 
of fat adipogenesis has also been reported (27). A recent study showed that BMAL1 silencing-induced BMP2 was found in rat uterus endometrium stromal cells (28). Samsa et al (5) recently reported that $\mathrm{BMAL1}^{-/-}$mice had reduced osteoblast differentiation in vitro and they proposed some potential molecular mechanism of BMAL1-dependent control of osteogenic differentiation including oxidative stress response, the mTOR signaling pathway and translation. However, the precise mechanism via which BMAL1 induces osteogenic differentiation of BMSCs remains unclear. In the present study, it was demonstrated that BMAL1 deficiency decreased BMP2 expression during osteogenic differentiation of BMSCs.

The disorder and downregulation of clock genes may be the cause of disease, and in some cases could also be used as a target of drug action to improve disease symptoms and treat disease. Recently, in order to alleviate circadian clock-related disease, there has been significant progress in the development of chemical compounds which are capable of manipulating clock genes as novel preventive and therapeutic agents (29). For example, resveratrol, which is considered a natural antioxidant polyphenol compound, has also been demonstrated to ameliorate circadian disorders of lipid metabolism (30). Moreover, a recent study revealed that glucocorticoids altered the circadian clock in the iris-ciliary body in a mouse model (31). However, few studies have yet demonstrated the potential mechanism by which osteogenic differentiation is mediated between icariin and BMAL1.

The results of the present study suggested that icariin at a concentration range between $10^{-9}$ and $10^{-5} \mathrm{~mol} / \mathrm{l}$ significantly promoted the proliferation of BMSCs, especially at $10^{-7} \mathrm{~mol} / \mathrm{l}$. A number of previous studies have reported that icariin increased cell proliferation at different concentrations. Yang et al (32) showed enhanced proliferation of human neural stem cells in vitro at a concentration of $10^{-5} \mathrm{~mol} / \mathrm{l}$. In addition, Mok et al (33) demonstrated that icariin stimulated the proliferation of UMR 106 cells at concentrations ranging from $10^{-14}$ to $10^{-6} \mathrm{~mol} / \mathrm{l}$. These differences may be attributable to the variety of cells used in the experiments.

Icariin has been demonstrated to have therapeutic properties, with reports of antitumor, anti-hepatotoxic, immune-enhancing, anti-inflammatory, neurite outgrowth activity and erection dysfunction-improving effects over the past decades among Chinese researchers (34-36). Furthermore, the bone-strengthening activity of icariin has attracted much recent attention. However, there have been few reports on the effect of icariin on the circadian clock in BMSCs. The present study suggested that icariin increased BMAL1 expression. Furthermore, reduced expression of BMP2/RUNX2 was observed in the present study during osteogenic differentiation of BMAL1-deficient cells. In addition, mineralized nodule formation was suppressed both in BMAL1 ${ }^{-/}$BMSCs and in icariin-mediated BMAL1 ${ }^{-/-}$BMSCs. Taken together; these findings demonstrated that icariin promoted osteogenic differentiation by upregulating BMAL1 expression through BMP signaling in BMSCs. However, as the effects of icariin on BMSCs in the present study were mainly observed in vitro, further studies will be needed to confirm these results in animal models.

In conclusion, to the best of our knowledge, the present study is the first to provide evidence that icariin promoted osteogenic differentiation through BMAL1-BMP2/RUNX2 signaling.

\section{Acknowledgements}

Not applicable.

\section{Funding}

The present study was supported by the Natural Science Foundation of Hubei Province (grant. nos. 2013CFB376 and 2018CFB095).

\section{Availability of data and materials}

The datasets used during the present study are available from the corresponding author upon reasonable request.

\section{Authors' contributions}

ZH and SZ conceived and designed the study. HW, ZL, JX and $\mathrm{ZW}$ performed the experiments. $\mathrm{ZH}$ and $\mathrm{HW}$ wrote the paper. XW, YH, YX and XL analyzed the data, and reviewed and edited the manuscript. All authors read and approved the manuscript and agree to be accountable for all aspects of the research.

\section{Ethics approval and consent to participate}

All the experimental protocols involving animals were approved by the Institutional Animal Care and Use Committee of Tongji Medical College, Huazhong University of Science and Technology (IACUU no. S496; date of permission, October, 31, 2017).

\section{Patient consent for publication}

Not applicable.

\section{Competing interests}

The authors declare they have no competing interests.

\section{References}

1. Mankin HJ: Nontraumatic necrosis of bone (osteonecrosis). N Engl J Med 326: 1473-1479, 1992.

2. Yu Y, Zhang Y, Wu J, Sun Y, Xiong Z, Niu F, Lei L, Du S, Chen P and Yang Z: Genetic polymorphisms in IL1B predict susceptibility to steroid-induced osteonecrosis of the femoral head in Chinese Han population. Osteoporos Int 30: 871-877, 2019.

3. Mont MA, Cherian JJ, Sierra RJ, Jones LC and Lieberman JR: Nontraumatic osteonecrosis of the femoral head: Where do we stand today? A ten-year update. J Bone Joint Surg Am 97: 1604-1627, 2015

4. Shui C, Spelsberg TC, Riggs BL and Khosla S: Changes in Runx2/Cbfa1 expression and activity during osteoblastic differentiation of human bone marrow stromal cells. J Bone Miner Res 18: 213-221, 2003.

5. Samsa WE, Vasanji A, Midura RJ and Kondratov RV: Deficiency of circadian clock protein BMAL1 in mice results in a low bone mass phenotype. Bone 84: 194-203, 2016.

6. He Y, Chen Y, Zhao Q and Tan Z: Roles of brain and muscle ARNT-like 1 and Wnt antagonist Dkk1 during osteogenesis of bone marrow stromal cells. Cell Prolif 46: 644-653, 2013. 
7. Mohawk JA, Green CB and Takahashi JS: Central and peripheral circadian clocks in mammals. Annu Rev Neurosci 35: 445-462, 2012

8. Richards J and Gumz ML: Mechanism of the circadian clock in physiology. Am J Physiol Regul Integr Comp Physiol 304: R1053-R1064, 2013.

9. DeBruyne JP, Weaver DR and Dallmann R: The hepatic circadian clock modulates xenobiotic metabolism in mice. J Biol Rhythms 29: 277-287, 2014.

10. Zmrzljak UP and Rozman D: Circadian regulation of the hepatic endobiotic and xenobitoic detoxification pathways: The time matters. Chem Res Toxicol 25: 811-824, 2012.

11. Bailey SM, Udoh US and Young ME: Circadian regulation of metabolism. J Endocrinol 222: R75-R96, 2014.

12. Miranda J, Portillo MP, Madrid JA, Arias N, Macarulla MT and Garaulet M: Effects of resveratrol on changes induced by high-fat feeding on clock genes in rats. Br J Nutr 110: 1421-1428, 2013.

13. Chen P, Kakan X and Zhang J: Altered circadian rhythm of the clock genes in fibrotic livers induced by carbon tetrachloride. FEBS Lett 584: 1597-1601, 2010.

14. Gabás-Rivera C, Martínez-Beamonte R, Ríos JL, Navarro MA, Surra JC, Arnal C, Rodríguez-Yoldi MJ and Osada J: Dietary oleanolic acid mediates circadian clock gene expression in liver independently of diet and animal model but requires apolipoprotein A1. J Nutr Biochem 24: 2100-2109, 2013.

15. Huang Z, Cheng C, Wang J, Liu X, Wei H, Han Y, Yang S and Wang $\mathrm{X}$ : Icariin regulates the osteoblast differentiation and cell proliferation of MC3T3-E1 cells through microRNA-153 by targeting Runt-related transcription factor 2. Exp Ther Med 15: 5159-5166, 2018

16. Huang Z, Cheng C, Cao B, Wang J, Wei H, Liu X, Han Y, Yang S and Wang $X$ : Icariin protects against glucocorticoid-induced osteonecrosis of the femoral head in rats. Cell Physiol Biochem 47: 694-706, 2018.

17. Gong H, Wang X, Wang L, Liu Y, Wang QL, Pang H, Zhang Q, Wang Z: Inhibition of IGF-1 receptor kinase blocks the differentiation into cardiomyocyte-like cells of BMSCs induced by IGF-1. Molecular medicine reports 16: 787-793, 2017.

18. Livak KJ and Schmittgen TD: Analysis of relative gene expression data using real-time quantitative PCR and the 2(-Delta Delta C(T)) method. Methods 25: 402-408, 2001.

19. Dierickx P, Van Laake LW and Geijsen N: Circadian clocks: From stem cells to tissue homeostasis and regeneration. EMBO Rep 19: 18-28, 2018

20. Murray IR, West CC, Hardy WR, James AW, Park TS, Nguyen A, Tawonsawatruk T, Lazzari L, Soo C and Péault B: Natural history of mesenchymal stem cells, from vessel walls to culture vessels. Cell Mol Life Sci 71: 1353-1374, 2014.

21. Weger M, Diotel N, Dorsemans AC, Dickmeis $T$ and Weger BD: Stem cells and the circadian clock. Dev Biol 431: 111-123, 2017.

22. Fu L, Patel MS, Bradley A, Wagner EF and Karsenty G: The molecular clock mediates leptin-regulated bone formation. Cell 122: 803-815, 2005.

23. Gosselet FP, Magnaldo T, Culerrier RM, Sarasin A and Ehrhart JC: BMP2 and BMP6 control p57(Kip2) expression and cell growth arrest/terminal differentiation in normal primary human epidermal keratinocytes. Cell Signal 19: 731-739, 2007.
24. Rogers MB, Shah TA and Shaikh NN: Turning bone morphogenetic protein 2 (BMP2) on and off in mesenchymal cells. J Cell Biochem 116: 2127-2138, 2015.

25. Shu B, Zhang M, Xie R, Wang M, Jin H, Hou W, Tang D, Harris SE, Mishina Y, O'Keefe RJ, et al: BMP2, but not BMP4, is crucial for chondrocyte proliferation and maturation during endochondral bone development. J Cell Sci 124: 3428-3440, 2011.

26. Hirai T, Tanaka K and Togari A: $\alpha 1$-adrenergic receptor signaling in osteoblasts regulates clock genes and bone morphogenetic protein 4 expression through up-regulation of the transcriptional factor nuclear factor IL-3 (Nfil3)/E4 promoter-binding protein 4 (E4BP4). J Biol Chem 289: 17174-17183, 2014.

27. Nam D, Guo B, Chatterjee S, Chen MH, Nelson D, Yechoor VK and Ma K: The adipocyte clock controls brown adipogenesis through the TGF- $\beta$ and BMP signaling pathways. J Cell Sci 128: $1835-1847,2015$.

28. Tasaki H,Zhao L, Isayama K, Chen H, Yamauchi N, Shigeyoshi Y, Hashimoto S and Hattori MA: Inhibitory role of REV-ERB $\alpha$ in the expression of bone morphogenetic protein gene family in rat uterus endometrium stromal cells. Am J Physiol Cell Physiol 308: C528-C538, 2015.

29. Gloston GF, Yoo SH and Chen ZJ: Clock-enhancing small molecules and potential applications in chronic diseases and aging. Front Neurol 8: 100, 2017.

30. Sun L, Wang Y, Song Y, Cheng XR, Xia S, Rahman MR, Shi Y and Le G: Resveratrol restores the circadian rhythmic disorder of lipid metabolism induced by high-fat diet in mice. Biochem Biophys Res Commun 458: 86-91, 2015.

31. Tsuchiya S, Sugiyama K and Van Gelder RN: Adrenal and glucocorticoid effects on the circadian rhythm of murine intraocular pressure. Invest Ophthalmol Vis Sci 59: 5641-5647, 2018.

32. Yang P, Guan YQ, Li YL, Zhang L, Zhang L and Li L: Icariin promotes cell proliferation and regulates gene expression in human neural stem cells in vitro. Mol Med Rep 14: 1316-1322, 2016.

33. Mok SK, Chen WF, Lai WP, Leung PC, Wang XL, Yao XS and Wong MS: Icariin protects against bone loss induced by oestrogen deficiency and activates oestrogen receptor-dependent osteoblastic functions in UMR 106 cells. Br J Pharmacol 159: 939-949, 2010.

34. Chen SR, Xu XZ, Wang YH, Chen JW, Xu SW, Gu LQ and Liu PQ: Icariin derivative inhibits inflammation through suppression of p38 mitogen-activated protein kinase and nuclear factor-kappaB pathways. Biol Pharm Bull 33: 1307-1313, 2010.

35. Xu CQ, Liu BJ, Wu JF, Xu YC, Duan XH, Cao YX and Dong JC: Icariin attenuates LPS-induced acute inflammatory responses: Involvement of PI3K/Akt and NF-kappaB signaling pathway. Eur J Pharmacol 642: 146-153, 2010.

36. Zhou J, Wu J, Chen X, Fortenbery N, Eksioglu E, Kodumudi KN, Pk EB, Dong J, Djeu JY and Wei S: Icariin and its derivative, ICT, exert anti-inflammatory, anti-tumor effects, and modulate myeloid derived suppressive cells (MDSCs) functions. Int Immunopharmacol 11: 890-898, 2011.

This work is licensed under a Creative Commons Attribution-NonCommercial-NoDerivatives 4.0 International (CC BY-NC-ND 4.0) License. 